\title{
TNF- $\alpha$ Induces a Pro-Inflammatory Phenotypic Shift in Monocytes through ACSL1: Relevance to Metabolic Inflammation
}

\author{
Fatema Al-Rashed ${ }^{a}$ Zunair Ahmad ${ }^{a, b}$ Mina Amin Iskandar ${ }^{a, b}$ \\ Jaakko Tuomilehtoc,d Fahd Al-Mulla ${ }^{a}$ Rasheed Ahmad ${ }^{a}$ \\ almmunology Unit, Dasman Diabetes Institute, Kuwait-City, Kuwait, bSchool of Medicine, Royal College \\ of Surgeons in Ireland, Medical University of Bahrain, Busaiteen, Bahrain, 'University of Helsinki, \\ Helsinki, Finland, dDanube University Krems, Krems, Austria
}

\section{Key Words}

Monocytes $\cdot \mathrm{ACSL} 1 \cdot \mathrm{TNF}-\alpha \cdot \mathrm{CD} 11 \mathrm{c} \cdot$ Inflammation

\begin{abstract}
Background/Aims: TNF- $\alpha$-mediated pro-inflammatory phenotypic change in monocytes is known to be implicated in the pathogenesis of metabolic inflammation and insulin resistance. However, the mechanism by which TNF- $\alpha$-induces inflammatory phenotypic shift in monocytes is poorly understood. Since long-chain acyl-CoA synthetase 1 (ACSL1) is associated with inflammatory monocytes/macrophages, we investigated the role of ACSL1 in the TNF- $\alpha$-driven inflammatory phenotypic shift in the monocytes. Methods: Monocytes (Human monocytic THP-1 cells) were stimulated with TNF- $\alpha$. Inflammatory phenotypic markers (CD16, CD11b, CD11c and HLA-DR) expression was determined with real time RT$P C R$ and flow cytometry. IL-1 $\beta$ and MCP-1 were determined by ELISA. Signaling pathways were identified by using ACSL1 inhibitor, ACSL1 siRNA and NF-KB reporter monocytic cells. Phosphorylation of NF-KB was analyzed by western blotting and flow cytometry. Results: Our data show that TNF- $\alpha$ induced significant increase in the expression of CD16, CD11b, CD11c and HLA-DR. Inhibition of ACSL1 activity in the cells with triacsin C significantly suppressed the expression of these inflammatory markers. Using ACSL-1 siRNA, we further demonstrate that TNF- $\alpha$-induced inflammatory markers expression in monocytic cells requires ACSL1. In addition, IL-1b and MCP-1 production by TNF- $\alpha$ activated monocytic cells was significantly blocked by the inhibition of ACSL-1 activity. Interestingly, elevated NF-KB activity resulting from TNF- $\alpha$ stimulation was attenuated in ACSL1 deficient cells. Conclusion: Our findings provide an evidence that TNF- $\alpha$-associated inflammatory polarization in monocytes is an ACSL1 dependent process, which indicates its central role in TNF- $\alpha$-driven metabolic inflammation.




\section{Cellular Physiology Cell Physiol Biochem 2019;52:397-407 \\ \begin{tabular}{ll|l} 
and Biochemistry & $\begin{array}{l}\text { DOl: } 10.33594 / 000000028 \\
\text { Published online: } 8 \text { March } 2019\end{array}$ & $\begin{array}{l}\text { C } 2019 \text { The Author(s). Published by } \\
\text { Cell Physiol Biochem Press GmbH\&Co. KG }\end{array}$
\end{tabular} \\ Al-Rashed et al.: TNF- $\alpha$ Activates Monocytes via ACSL1}

\section{Introduction}

Chronic low-grade inflammation is a central characteristic of obesity and is involved in the development of insulin resistance in adipose tissue, muscle, and liver. This is caused by inflammatory mediators produced by activated circulatory and infiltrated immune cells in adipose tissue. Among the immune cells, monocytes/macrophages play an important role in obesity-induced adipose tissue inflammation. Monocytes (precursor cells of macrophages) isolated from obese humans display an inflammatory phenotype and secrete higher levels of pro-inflammatory cytokines/chemokines such as TNF- $\alpha$, IL-6, IL-1b, and MCP- 1 compared to monocytes from the lean individuals $[1,2]$. Distinct monocyte subsets could be identified based on the expression of the surface antigen CD16. Classical monocytes do not express the CD16 antigen (CD14++CD16-), while "non-classical" monocytes express CD16 on the cell surface (CD14+CD16++). The expression pattern of the two surface markers reflect the function of the monocytes. It is well noted that monocytes/macrophages in obesity are polarized to more pro-inflammatory phenotype (M1). The primary trigger for the recruitment of monocytes into the adipose tissue is thought to be the secretion of the chemokine; Monocyte chemoattractant protein-1 (MCP-1) by TNF- $\alpha$-activated enlarged adipocytes. Pro-inflammatory monocytes/macrophages have high surface expression markers like CD14, CD16, CD11b, CD11c, and HLA-Dr [3, 4].

Increased circulating levels of TNF- $\alpha$ have been found in obese humans and rodents [5]. TNF- $\alpha$ action in obesity has been considered mainly inflammatory because it activates immune cells particularly monocytes and macrophages [6]. TNF- $\alpha$ plays a key role in shifting monocytes/macrophages into inflammatory state. However, the mechanism by which TNF- $\alpha$ induces this pro-inflammatory shift remains elusive. In obesity and type 2 diabetes (T2D), plasma and tissue levels of free fatty acids (FFAs) are elevated and have been suggested to contribute to the development of chronic inflammation and insulin resistance [7]. Since acyl-CoA synthetase 1 (ACSL1), which converts FFAs into their acyl-CoA derivatives, is involved in promoting inflammatory phenotype of the monocytes/macrophages associated diabetes [8], we hypothesized that ACSL1 could play a key role in the TNF- $\alpha$ mediated inflammatory polarization in monocytes. Here we show that blocking ACSL1 activity inhibits the expression of inflammatory markers in monocytes. ACSL1 deficient cells express less inflammatory markers in response to TNF- $\alpha$. ACSL1 specific siRNA and its inhibitor also block the production of inflammatory mediators such as IL-1b and MCP-1. TNF- $\alpha$-mediated NF- $\kappa B$ activity was also blocked in ACSL1 deficient cells. Collectively, our data show an interesting novel role of ACSL1 in TNF- $\alpha$-driven inflammation.

\section{Materials and Methods}

\section{Cell Culture}

Monocytes, human monocytic THP-1 cells, were purchased from American Type Culture Collection (ATCC) and grown in RPMI-1640 culture medium (Gibco, Life Technologies, Grand Island, USA) supplemented with 10\% fetal bovine serum (Gibco, Life Technologies, Grand Island, NY, USA), 2 mM glutamine (Gibco, Invitrogen, Grand Island, NY, USA), $1 \mathrm{mM}$ sodium pyruvate, $10 \mathrm{mM} \mathrm{HEPES,} 100 \mathrm{ug} / \mathrm{ml}$ Normocin, $50 \mathrm{U} / \mathrm{ml}$ penicillin and $50 \mu \mathrm{g} / \mathrm{ml}$ streptomycin (P/S; (Gibco, Invitrogen, Grand Island, NY, USA), and incubation at $37^{\circ} \mathrm{C}$ (with humidity) in 5\% CO2. NF-кB reporter monocytic cells (THP-1-XBlue) stably expressing a secreted embryonic alkaline phosphatase (SEAP) reporter inducible by NF- $\kappa$ B were purchased from InvivoGen (InvivoGen, San Diego, CA, USA). THP-1-XBlueTM-defMyD cells (monocytes deficient in MyD88 activity or MyD88-/- monocytes) were purchased from InvivoGen (San Diego, CA, USA). THP-1-XBlue cells were cultured in complete RPMI medium with the addition of zeocin $(200 \mu \mathrm{g} / \mathrm{ml})$ to select for cells expressing the SEAP -NF- $\kappa$ B reporter (InvivoGen, San Diego, CA, USA). Prior to stimulation, monocytes were transferred to normal serum free medium and plated in 12-well plates (Costar, Corning Incorporated, Corning, NY, USA) at $1 \times 106$ cells/well cell density unless indicated otherwise. 


\section{Cellular Physiology Cell Physiol Biochem 2019;52:397-407 \\ \begin{tabular}{ll|l} 
and Biochemistry $10.33594 / 000000028$ & $\begin{array}{l}\text { C) } 2019 \text { The Author(s). Published by } \\
\text { Published online: } 8 \text { March } 2019\end{array}$ & \begin{tabular}{l} 
Cell Physiol Biochem Press GmbH\&Co. KG \\
\hline
\end{tabular}
\end{tabular}}

Al-Rashed et al.: TNF- $\alpha$ Activates Monocytes via ACSL1

\section{Cell stimulation}

Monocytes were plated in 12-well plates (Costar, Corning Incorporated, Corning, NY, USA) at $1 \times 106$ cells/well concentration unless indicated otherwise. Cells were stimulated with TNF- $\alpha$ (10ng/ml) or LPS $\left(10 \mathrm{ng} / \mathrm{ml}\right.$; Sigma, Saint Louis, MO, USA) or Vehicle $(0.1 \% \mathrm{BSA})$ for $6-12 \mathrm{hrs}$ at $37^{\circ} \mathrm{C}$. Cells were harvested for RNA isolation and staining and culture media were collected for measuring IL-1b and MCP-1 secretion.

\section{Real time quantitative RT-PCR}

Total RNA was extracted using RNeasy Mini Kit (Qiagen, Valencia. CA, USA) per the manufacturer's instructions. The cDNA was synthesized using $1 \mu \mathrm{g}$ of total RNA using high capacity cDNA reverse transcription kit (Applied Biosystems, Foster city, CA, USA). Real-time PCR was performed on 7500 Fast Real-Time PCR System (Applied Biosystems, Foster City, CA, USA) using TaqMan® Gene Expression Master Mix (Applied Biosystems, Foster city). Each reaction contained 500ng cDNA that was amplified with Inventoried TaqMan Gene Expression Assay products (ITGAM: Hs00355885_m1; TNF: Hs01113624_g1; GAPDH: Hs03929097_ g1; HLA-DR: Hs00219575_m1; ACSL1: Hs00960561_m1). The threshold cycle (Ct) values were normalized to the house-keeping gene GAPDH and the amounts of target mRNA relative to control were calculated with $\Delta \Delta \mathrm{Ct}$-method $[9,10]$. Relative mRNA expression was expressed as fold expression over average of control gene expression. The expression level in control treatment was assumed to be 1 . Values are presented as mean \pm SEM. Results were analyzed statistically; $\mathrm{P}<0.05$ was considered significant.

\section{Extracellular staining -flow cytometry}

After different treatments, monocytic cells (1x106 cells) were resuspended in FACS staining buffer (BD Biosciences) and blocked with human IgG (Sigma; 20 $\mu \mathrm{g}$ ) for 30 minutes on ice. Cells were washed and resuspended in $100 \mathrm{ul}$ of FACS buffer and incubated with CD16 (CB16)-APC (cat\# 17-0168-42 ;BD Biosciences), CD11b (D12)-APC (cat\# 340936 ;BD Biosciences), CD11b-FITC (cat\# IM0530U; Beckmen coulter), CD11c (S_HCL-3)-PE (cat\# 347637 ;BD Biosciences) or HLA-DR-PE (cat\# IM1639 ;Beckmen coulter) on ice for 30 minutes. Cells were washed three times with FACS buffer and resuspended in $2 \%$ paraformaldehyde. Cells were centrifuged and resuspended in FACS buffer for FACS analysis (FACSCanto II; BD Bioscience, San Jose, USA). FACS data analysis was performed using BD FACSDivaTM Software 8 (BD Biosciences, San Jose, USA)

\section{Small interfering RNA (siRNA) transfections}

Monocytes were washed and resuspended in $100 \mathrm{ul}$ of nucleofector solution provided with the Amaxa Noclecfector Kit V and transfected separately with siRNA-ACSL1 (30nM; OriGene Technologies, Inc. MD, USA), scramble (control) siRNA (30nM; OriGene Technologies, Inc. MD, USA, USA), and pmaxGFP (0.5 ug; Amaxa Noclecfector Kit V for THP-1, Lonza). All transfection experiments were performed with Amaxa Cell Line Nucleofector Kit V for monocytic cells(Lonza, Germany) by using Amaxa Electroporation System (Amaxa Inc, Germany) according to the manufacturer's protocol [11]. After 36 hours of transfection, cells were treated with TNF- $\alpha$ for 6-12 hours. Cells were harvested for RNA isolation and staining study. ACSL1 gene knock down level was assessed by Real Time-PCR using ACSL1 gene specific primer probes.

\section{$I L-1 b$ and MCP-1 determination}

Secreted IL-1b and MCP-1 protein in supernatants of monocytic cells stimulated with TNF- $\alpha$ was quantified using sandwich ELISA following the manufacturer's instructions (R\&D systems, Minneapolis, USA).

\section{Western blotting}

Monocytic cells treated with TNF- $\alpha$ at different time points were harvested and incubated for 30 minutes with lysis buffer (10X Lysis Buffer, Cell signaling, USA). The protein lysates were prepared and resolved by $12 \%$ SDS-PAGE as described earlier [12]. Cellular proteins were transferred to Immuno-Blot PVDF membrane (Bio-Rad Laboratories, USA) by electro blotting. The membranes were then blocked with $5 \%$ non-fat milk in PBS for 1 hour, followed by incubation with primary antibodies against p-NF-кB and NF- $\mathrm{B}$ in 1: 1000 dilution at $4^{\circ} \mathrm{C}$ overnight. All the primary antibodies were purchased from Cell Signalling (Cell Signalling Technology, Inc). The blots were then washed three times with TBS-T and incubated for 2 hours with HRP-conjugated secondary antibody (Promega, Madison, WI, USA). Immunoreactive bands 
were developed using an Amersham ECL Plus Western Blotting Detection System (GE Health Care, Buckinghamshire, UK) and visualized by Molecular Imager ${ }^{\circledR}$ VersaDocTM MP Imaging Systems (Bio-Rad Laboratories, Hercules, CA, USA).

\section{Measurement of $N F-\kappa B$ activity}

NF-кB reporter monocytes (THP-1 XBlue; InvivoGen, San Diego, CA) are stably transfected with a reporter construct, expressing a secreted embryonic alkaline phosphatase (SEAP) gene under the control of a promoter inducible by the transcription factors NF- $\kappa$ B. Upon stimulation, NF- $\kappa B$ is activated and subsequently the secretion of SEAP is stimulated. Cells were stimulated with TNF- $\alpha$ (10 ng/ml) for 6-12 hours at $37^{\circ} \mathrm{C}$. Levels of SEAP were detected in the culture media after 3 hours incubation of supernatants with Quanti-Blue solution (InvivoGen, San Diego, CA, USA) at 650nm wave length by ELISA reader [13].

\section{Statistical Analysis}

Statistical analysis was performed using GraphPad Prism software (La Jolla, CA, USA). Data are shown as mean \pm standard error of the mean, unless otherwise indicated. Unpaired Student t-test and one way ANOVA followed by tukey's test were used to compare means between groups. For all analyses, data from a minimum of three sample sets were used for statistical calculation. P value $<0.05$ was considered significant. Ns: no significance, ${ }^{*} \mathrm{P}<0.05,{ }^{* *} \mathrm{P}<0.01,{ }^{* * *} \mathrm{P}<0.001$ and ${ }^{* * * *} \mathrm{P}<0.0001$ )

\section{Results}

\section{Inhibition of ACSL1 suppresses the TNF- $\alpha$ induced pro-inflammatory phenotypic shift in} monocytes

To query whether ACSL1 was required for TNF- $\alpha$-mediated activation of monocytes, we used triacsin $c$ to inhibit ACSL1 expression in monocytes (Fig. 1A) and measured the phenotypic inflammatory markers expression in these cells. Our data show that pretreatment of the monocytes with triacsin $c$, followed by the exposure to TNF- $\alpha$, caused a significant suppression in the expression of inflammatory marker CD16 (Fig. 1B and C). In the case of obesity setting, CD11c is abundantly expressed in monocytes and macrophages [14]. We found that TNF- $\alpha$ upregulates the expression of CD11c at both mRNA and protein levels in monocyes. Inhibition of ACSL1 significantly blocks the expression of CD11c in monocyes (Fig. 1D-F). Since CD11b and HLA-DR cell surface are considered phenotypic markers for inflammatory monocytes, we also found that TNF- $\alpha$-induced CD11b and HLA-DR expression in monocytes were blocked by inhibition of ACSL1 (Fig. 1G-L).

Activated monocytes/macrophages play a role in the pathogenesis of the different diseases by secreting inflammatory cytokines. Therefore, we questioned whether TNF- $\alpha$ mediated activation of monocytes led to the production of IL-1b and MCP-1. Our data showed that monocytes activated by TNF- $\alpha$ secreted IL-1b and MCP-1 in the culture media. Next, we asked whether this production of IL-1b and MCP-1 was reduced by the inhibition of ACSL1. Our data showed that the inhibition of ACSL1 activity in monocytes by triacsin c diminished the secretion of IL-1b and MCP-1 (Fig. 2A and B).

\section{ACSL1 deficiency inhibits TNF- $\alpha$ mediated pro-inflammatory shift in monocytes}

To further verify TNF- $\alpha$-induced inflammatory shift in the monocytes was dependent on ACSL1, we transfected cells with ACSL1 siRNA (siACSL1), which achieved more than 50\% reduction in ACSL1 mRNA levels compared with scramble (control) siRNA (Neg-siRNA) (Fig. 3A). Accordingly, the expression of surface CD16 protein marker was significantly reduced in ACSL1 siRNA-transfected cells after stimulation with TNF- $\alpha$ as compared with scramble siRNA-transfected cells (Fig. 3B and C). We also noted that CD11c mRNA and protein expression was significantly suppressed $(\mathrm{P}<0.05)$ in ACSL1 deficient cells activated with TNF- $\alpha$ (Fig. 3D-F). Similarly, CD11b and HLA-DR expression was not upregulated at both mRNA and protein levels in ACSL1 deficient cells when activated with TNF- $\alpha$ (CD11b: Fig. 2G-I; HLA-DR: Fig. 2J-L). 
We additionally wanted to know whether TNF- $\alpha$ mediated inflammatory effect on monocytes was due to the modulation in the expression of ACSL1. In this regard, the cells treated with TNF- $\alpha$ show high expression of ACSL1 as compared to the controls (Fig. 4A). ACSL1 siRNA transfection suppresses the endogenously as well as induced ACSL-1 expression in monocytic cells (Fig. 4B).

Altogether, our results show that ACSL1 is a key effector of inflammatory actions mediated by TNF- $\alpha$ in monocytic cells.

\section{Involvement of ACSL1 in} the TNF- $\alpha$ mediated NF-

$\kappa B$ activation

TNF- $\alpha$ was shown to regulate lipid metabolism through increasing free fatty acids production. This process occurs through both de-novo and exogenous pathways. The increase of intracellular concentration of fatty acid products such as long chain acyl-CoA (LCAC) have been shown to induce inflammation through $\mathrm{NF}-\kappa \mathrm{B}$ activation [15]. Therefore, we questioned whether ACSL1 is involved in the TNF- $\alpha$ mediated activation of NF$\kappa \mathrm{B}$. Monocytes treatment with TNF- $\alpha$ increased phosphorylation of NF-кB and it was reduced by the by treatment with inhibitor of ACSL1 (triacsin c) (Fig. 5A and B). Flow cytometric analysis of phosphorylated NF- $\kappa B$ P65 versus its inhibitor protein $\mathrm{IkB} \alpha$ indicates that, when monocytes were stimulated with TNF- $\alpha, 75.7 \%$ of cells

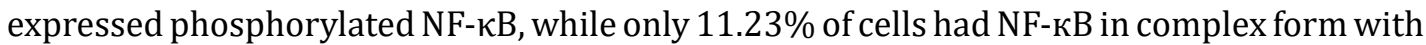
$\mathrm{IkB} \alpha$ (Fig. 5C). In contrast pre-treatment of the cells with triacsin c, blocked the translocation of NF- $\kappa$ B to the nucleus, where only $5.2 \%$ cells showed positive staining for $\mathrm{p}-\mathrm{NF}-\kappa \mathrm{B}$, while

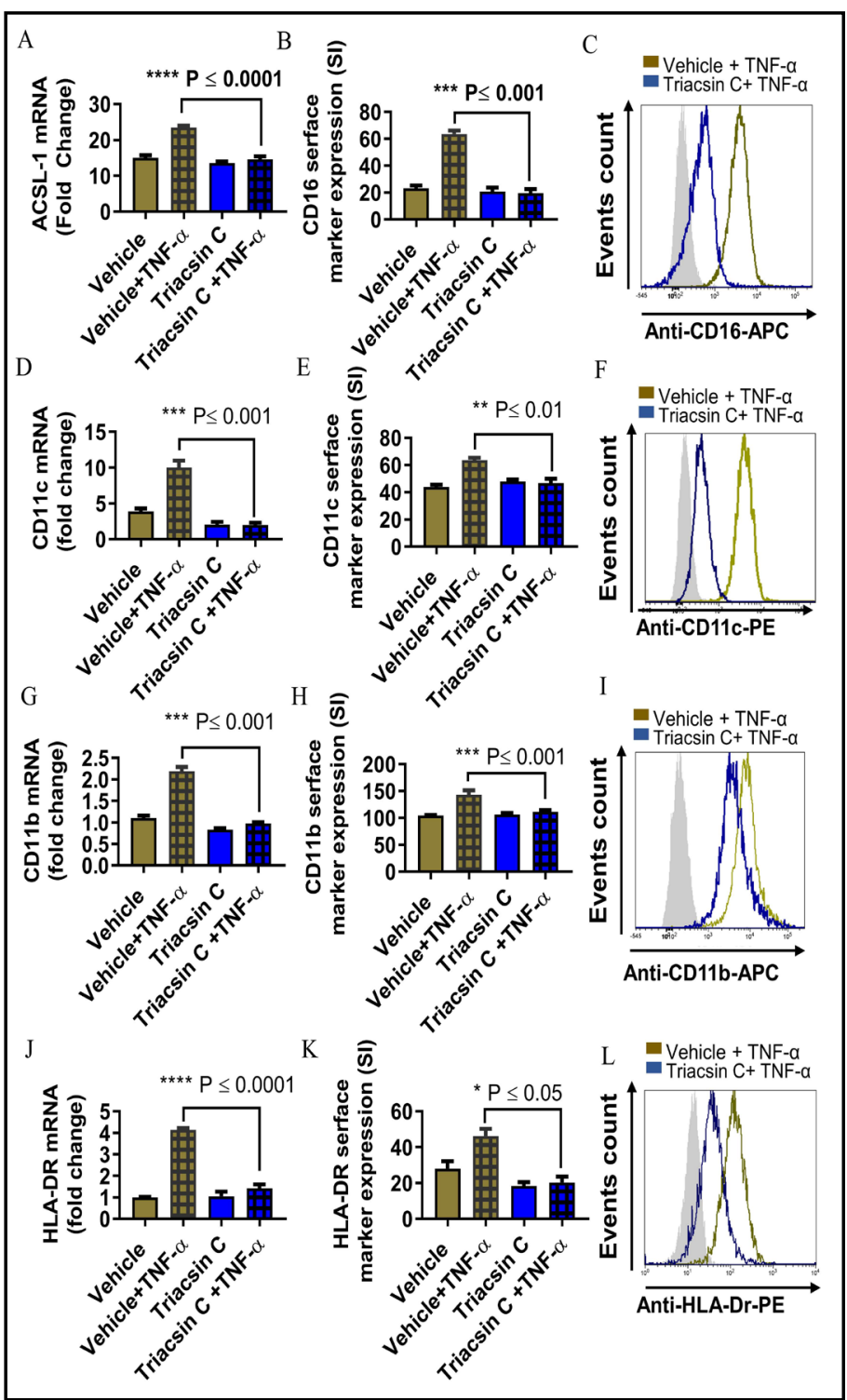

Fig. 1. ACSL1 inhibition blocks TNF- $\alpha$ mediated pro-inflammatory change in monocytes. Monocytic cells were pretreated with ACSL1 inhibitor (Triacsin C: $(1 \mathrm{uM})$ or vehicle for 1 hour and then incubated with TNF- $\alpha$ for 24 hours. (A, D, G, J) ACSL, CD11c, CD11b and HLADR mRNA were determined by real time RT-PCR. Triacsin C inhibted Cells were stained with antibody against CD16, CD11c, CD11b, or HLA-DR, along with matched control antibodies and assessed by flow cytometry. (B, E, H, K) Flow cytometry data are presented as a bar graph of mean staining intensity (SI) and (C, F, I, L) representative histogram. Bar graphs depict mean values \pm SEM of staining intensity (SI). $\mathrm{P}<0.05$ was considered as statistically significant $(* \mathrm{P}<0.01$; $\left.{ }^{* *} \mathrm{P}<0.001,{ }^{* * *} \mathrm{P}<0.0001\right)$. The data in all figures are representative of three independent experiments. 
the $76.2 \%$ cells had NF-кB in a complex form with IkB $\alpha$ (Fig. 5C). To further confirm the role of ACSL1 in TNF- $\alpha$ mediated activation of NF- $\kappa B$, we used NF$\kappa \mathrm{B}$ activity reporter monocytic cells. Our data show that TNF- $\alpha$ induces higher NF- $\kappa B$ activity in the NF$\kappa B$ reporter cells and this activity was not seen in the cells treated with ACSL1 inhibitor (triacsin c) (Fig. 5D).

To further verify the role of ACSL1 in NF- $\mathrm{KB}$ activation in TNF- $\alpha$ treated monocytes, ACSL1 deficient monocytes were generated by using siRNA approach. NF-кB phosphorylation effects of TNF- $\alpha$ were decreased in ACSL1 deficient cells (Fig. 5E and F). Flow cytometric analysis of phosphorylated NF$\kappa \mathrm{P} 65$ versus its inhibitor protein $\mathrm{IkB} \alpha$ indicates that, when ACSL1 deficient cells were stimulated with TNF- $\alpha, 9.9 \%$ of cells expressed positive staining for phosphorylated NF- $\mathrm{B}$, while $88.8 \%$ cells had NF-KB complexed with IkB $\alpha$ (Fig. 5F).

\section{Discussion}

Obesity-induced low grading chronic inflammation, a leading cause of insulin resistance, is characterized by more activation and infiltration of immune cells in peripheral tissues. Among the immune cells, monocytes play an important role in obesity-induced inflammation and they are divided into two classes based on their phenotype and function: inflammatory non-classical (M1) and classical (M2). The shift in the activation state of monocytes from classical (M2) to non-classical activated monocytes (M1) is enhanced in obesity. These activated monocytes subsequently infiltrate and differentiate into M1 macrophages within the adipose tissue in response to inflammatory cytokines $[16,17]$. It is well documented that elevated levels of TNF- $\alpha$ in obese humans and rodents influence enormously the phenotypic changes occurring in monocytes and, also associated with metabolic dysfunction and free fatty acids secretion by adipocytes. However, the mechanism by which

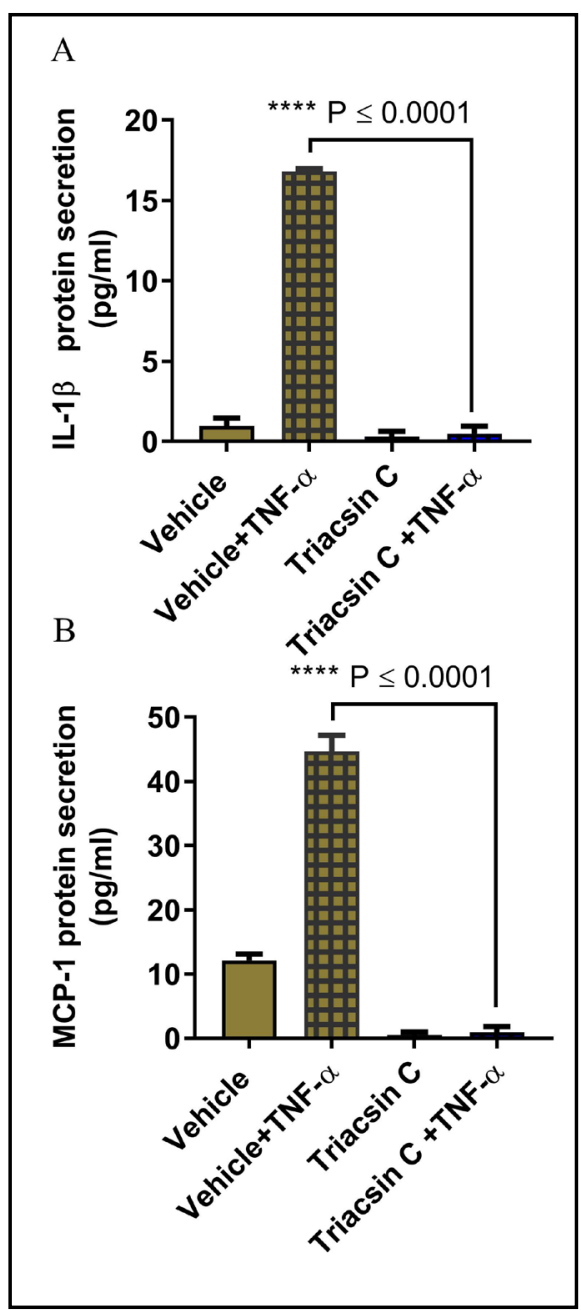

Fig. 2. IL-1b and MCP-1 secreted by TNF- $\alpha$ activated monocytes are suppressed by ACSL1 inhibition. (A and B) Monocytes were pretreated with ACSL1 inhibitor (triacsin C: $(1 \mathrm{uM})$ or vehicle for 1 hour and then incubated with TNF- $\alpha$ for 24 hours. Secreted IL-1b and MCP-1 protein in culture media was determined by ELISA. All data are expressed as mean \pm SEM ( $\mathrm{n} \geq$ 3). ${ }^{*} \mathrm{p}<0.05,{ }^{* *} \mathrm{p}<0.01,{ }^{* * *} \mathrm{p}<0.001$ versus vehicle. TNF- $\alpha$-induces the inflammatory phenotypic shift in monocytes remains elusive. In this study we report that long-chain acyl-CoA synthetase 1 (ACSL1), a key enzyme of lipid/fatty acids metabolism, is involved in TNF- $\alpha$-mediated proinflammatory phenotypic shift in monocytes. Our results show that blocking the activity of the ACSL1 by triacsin c inhibits the TNF- $\alpha$ induced CD16 expression in monocytes. Furthermore, ACSL1 deficiency prevents TNF- $\alpha$-induced inflammatory switch in monocytes, supporting the notion that inflammation induced by TNF- $\alpha$ is reduced by ACSL1 deficiency. CD16 expression in monocytes is increased in various inflammatory disorders such as coronary artery disease, asthma, infection, obesity, and T2D. CD16+ monocytes actively secrete inflammatory cytokines $[3,18]$. We further explored the impact of ACSL1 on the induction of CD11c marker, which is highly expressed on inflammatory monocytes and macrophages in 
Fig. 3. TNF- $\alpha$ mediated pro - in fla m matory phenotypic shift requires ACSL1. (A) Monocytes were transfected with NegsiRNA or siACSL1 siRNA and incubated for 36 hours. Real-time PCR was performed to measure ACSL1 expression. ACSL1deficient cells were treated with TNF- $\alpha$ and vehicle. (D, G, J) CD11c, CD11b, HLA-DR mRNA was determined by real time RT-PCR. Cells were stained with antibody against CD16, CD11c, CD11b, or HLA-DR, along with matched control antibody, and assessed by flow cytometry. (B, E, H, K). Flow cytometry data are presented as a bar graph of mean staining intensity (SI) as well as (C, F, I, L) representative histogram. Bar graphs depict mean values \pm SEM of staining intensity (SI). $\quad \mathrm{P}<0.05$ was considered as statistically significant (* $\mathrm{P}<0.01 ;{ }^{* *} \mathrm{P}<0.001$, *** $\mathrm{P}<$ 0.0001). The data in all figures are representative of three independent experiments.
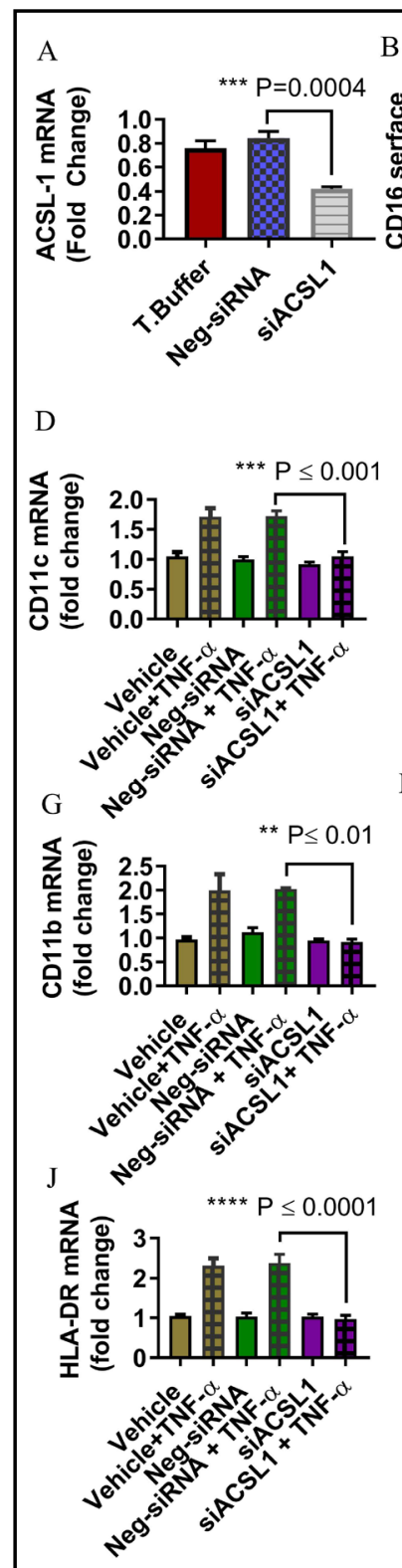
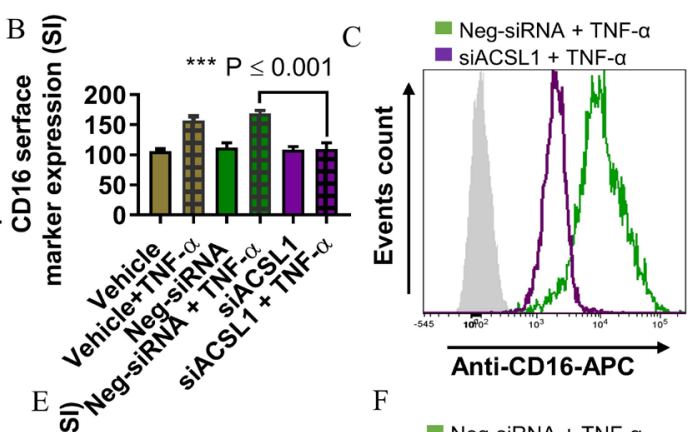

Neg-siRNA + TNF- $\alpha$

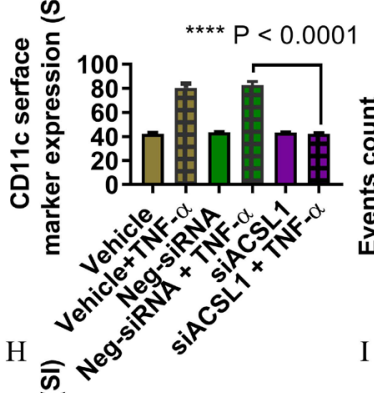

siACSL $1+$ TNF- $\alpha$

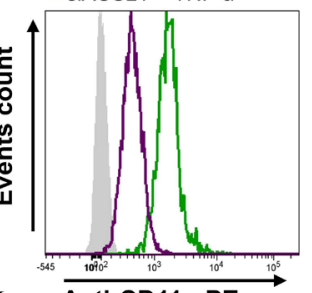

I

Anti-CD11c-PE

Neg-siRNA + TNF- $\alpha$

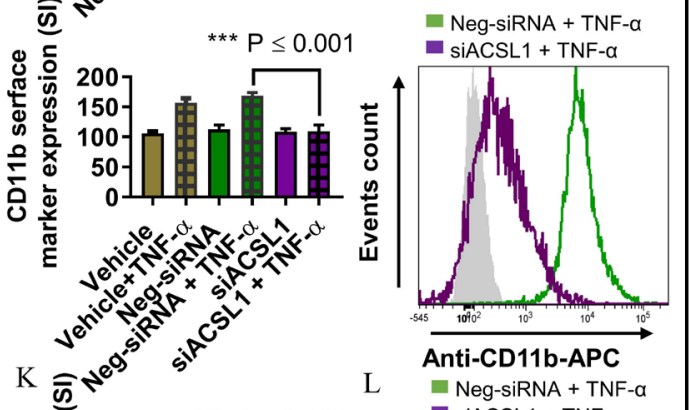

$\mathrm{L} \quad \mathrm{Neg}-\mathrm{SiRNA}+\mathrm{TNF}-\mathrm{a}$
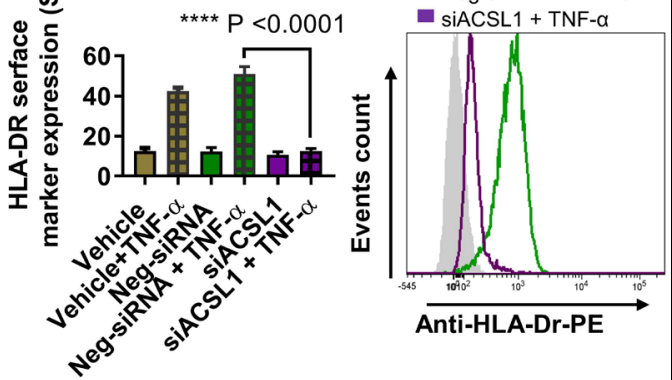

obesity setting. We found that interruption of ACSL1 in monocytes diminished the expression of CD11c in these cells induced by TNF- $\alpha$. We also observed the impact of ACSL1 on the expression of CD11b and HLA-DR in activated monocytes. It has been reported that mice fed on high fat diets had high numbers of CD11c+ monocytes/macrophages, suggesting that in obesity state, TNF- $\alpha$ modulates the phenotype of the monocytes/macrophages. Indeed, phenotypic switch of monocytes/macrophages in the adipose tissue is a central element of metabolic inflammation and insulin resistance in obese mice. CD16+ and CD11c+ monocytes are actively involved in the production of inflammatory cytokines and chemokines including IL-1b, TNF- $\alpha$, and MCP-1, pointing to increased migration of the inflammatory monocytes from the circulation into adipose tissue in obesity $[14,19]$ Our data show that the secretion of the IL-1b and MCP- 1 by the TNF- $\alpha$-treated monocytes was significantly blocked by the 
inhibition of ACSL1. Similarly, ACSL1 deficient cells do not show response to TNF- $\alpha$ for the production of IL$1 \mathrm{~b}$ and MCP-1. In the case of obesity, IL-1b and MCP1 expression appears to be essential for phenotypic and functional M1 polarization of monocytes/ macrophages in response to TNF- $\alpha$. Expression of CD16, CD11b, and CD11c correlates with expression of IL-1b and MCP1 in inflammatory monocytes. It is becoming increasingly clear that monocytes isolated from obese humans with diabetes display an inflammatory phenotype and secrete higher levels of pro-inflammatory mediators such as IL-6, MCP-1, and IL-1 $\beta$. Furthermore, inflammatory monocytes found in the setting of obesity and T2D have been shown as a result of cytokines/chemokines genes activation and metabolic dysfunction. Metabolic dysfunction is implicated in a wide-ranging effect on the immune cells and release of free fatty acids by adipocytes. Free fatty acids exert inflammatory effects in macrophages, which could contribute to inflammation in the setting of diabetes and associated complications. After entering the cell, these fatty acids are thioesterified into their acyl-CoA derivatives, a process catalyzed by long-chain acyl-CoA synthetases (ACSLs). It has been reported that diabetes promotes an inflammatory macrophage phenotype through ACSL1. Increased expression of ACSL1 is noted in macrophages from obese mice and rodents. ACSL1 is upregulated by LPS and TNF- $\alpha$; both are elevated in obesity [8]. We also found that ACSL1 expression is high in TNF- $\alpha$ activated inflammatory monocytes.

The transcription factor NF- $\kappa \mathrm{B}$ induces the expression of various pro-inflammatory genes, including those encoding cytokines and chemokines. Deregulated NF- $\kappa B$ activation contributes to the pathogenic processes of various inflammatory diseases. NF- $\mathrm{KB}$ is involved in polarization and differentiation of monocytes into activated state implicated in the development of inflammatory metabolic diseases [20]. Our results showed that deficiency of ACSL1 inhibits the phosphorylation and

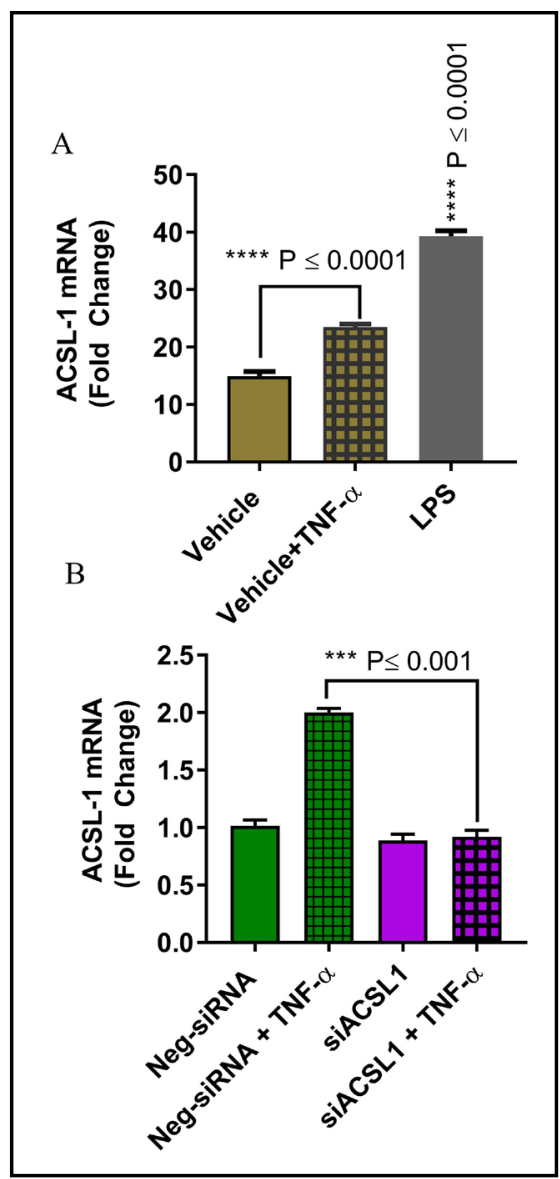

Fig. 4. Expression level of ACSL1 is increased TNF- $\alpha$ mediated proinflammatory monocytes. (A) Cells were incubated with TNF- $\alpha$ and vehicle for 24 hours. Cells were harvested and ACSL1 mRNA was determined by real time RTPCR. (B) ACSL-1 deficient monocytic cells were treated with TNF- $\alpha$ and vehicle for 24 hours. ACSL1 expression was determined by real time RT-PCR. The data in all figures are representative of three independent experiments. activation of NF- $\kappa B$ in response to TNF- $\alpha$, indicating a very interesting role of ACSL in TNF- $\alpha$ mediated activation of major transcription factor NF$к B$. These results indicate that ACSL1 acts upstream of the NF-кB pathway. These findings are consistent with the previous reports indicating that free fatty acid palmitate induces proinflammatory cytokine IL-6 production by increasing phosphorylation and transcriptional activity of NF- $\mathrm{BB}$ protein [21]. It has been reported that siRNA for ACSL3 inhibits palmitateinduced phosphorylation of p65 subunit of NF- $\kappa B$ and ACSL3 acts upstream of the NF- $\kappa B$ pathway. Palmitoyl-CoA or further metabolites of palmitoyl-CoA activates NF- $\kappa B$ signaling pathway through the activation of ACSL3 [22]. We further showed that ACSL1 is required in TNF- $\alpha$-driven production of IL-1b and MCP-1. Our results suggested that ACSL1 plays a key role in the inflammation induced by TNF- $\alpha$-activated monocytes. 
A

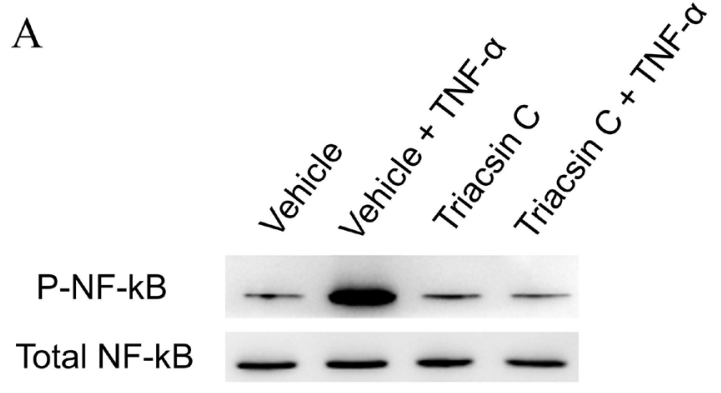

B

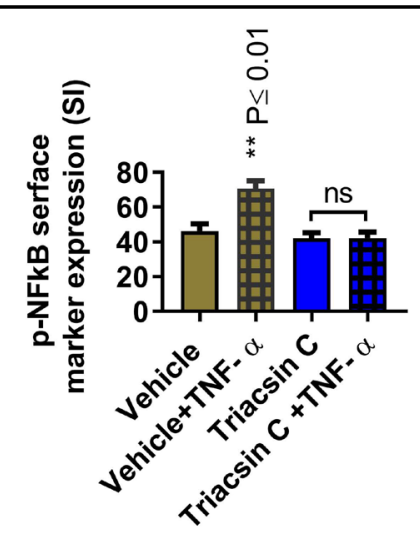

$\mathrm{E}$

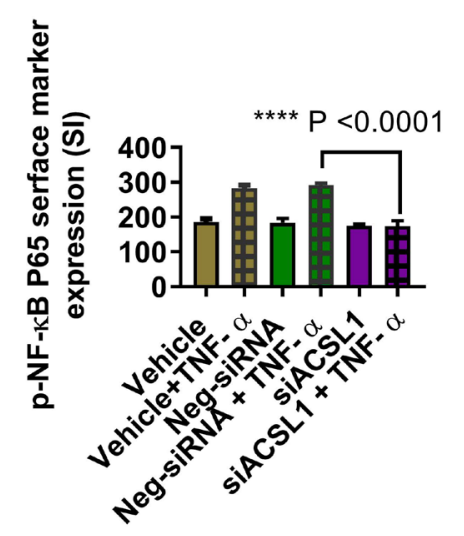

$\mathrm{F}$

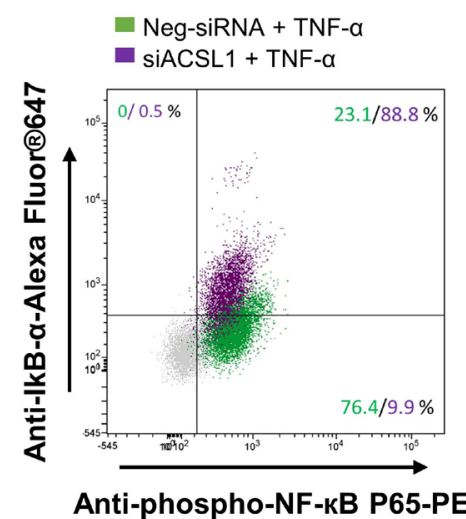

$\mathrm{D}$

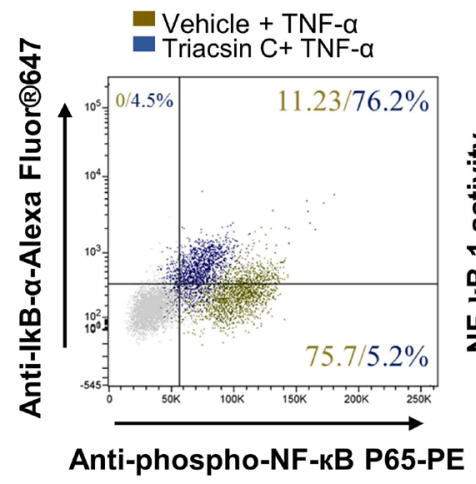

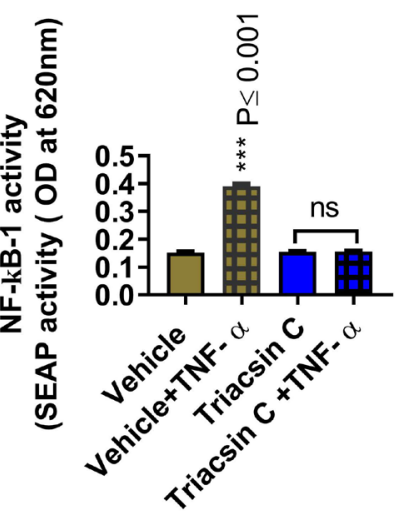

G

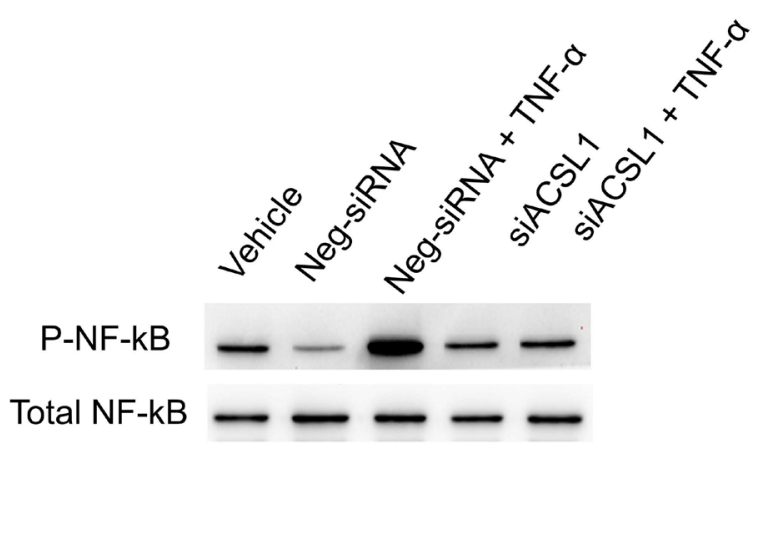

Fig. 5. NF-KB activation in TNF- $\alpha$ treated monocytes was inhibited by triacsin C or ACSL1 deficiency. Monocytic cells were pretreated with ACSL1 inhibitor (Triacsin C: (1uM) or vehicle for $1 \mathrm{hr}$ and then incubated with TNF- $\alpha$ for 15 minutes. NF-KB phosphorylation was determined by (A) western blotting and (B) flow cytometry. (C) Representative flow cytometry dot plots of p-NF-кB fluorescence versus total IkB $\alpha$ cells. (D) NF-кB reporter monocytic cells were pretreated with ACSL1 inhibitor (triacsin C: (1uM) or vehicle for 1 hour and then incubated with TNF- $\alpha$ for 24 hours. Cell culture media were assayed for SEAP reporter activity (degree of NF- $\mathrm{KB}$ activation). (E) ACSL-1 deficient cells were treated with TNF- $\alpha$ for 15 minutes. NF-KB phosphorylation was determined by (E) western blotting and (F) flow cytometry. (G) Representative flow cytometry dot plots of p-NF-кB fluorescence versus total IkB $\alpha$ cells. All data are expressed as mean \pm SEM $(\mathrm{n} \geq 3) .{ }^{*} \mathrm{p}<0.05,{ }^{* *} \mathrm{p}<0.01,{ }^{* * *} \mathrm{p}<0.001$ versus TNF- $\alpha$. 


\section{Conclusion}

In summary, the results of the present study identify ACSL1 as a relevant contributor to the TNF- $\alpha$-driven pro-inflammatory phenotypic polarization in monocytes. These findings could also provide an interesting pathophysiological novel connection between a key enzyme of fatty acids metabolism ACSL1 and TNF- $\alpha$ in obesity settings.

\section{Abbreviations}

ACSL1 (Acyl-CoA synthetase 1); BSA (Bovine serum albumin); FFAs (Free fatty acids); HLA-Dr (Human Leukocyte Antigen - DR isotype); IL-1b (Interleukin 1 beta); IL-6 (Interleukin 6); LPS (Lipopolysaccharide); LCAC (Long chain acyl-CoA); MCP-1/CCL2

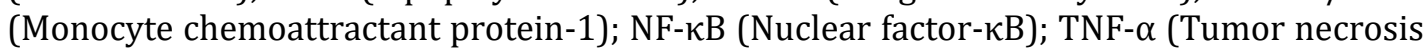
factor alpha); T1D (Type 1 diabetes); T2D (Type 2 diabetes).

\section{Acknowledgements}

This study was supported by Kuwait Foundation for Advancement of Sciences (KFAS) (Grant \#: RC-14016001, RA-2013-002, RA AM 2016-007), and L'Oréal-UNESCO FWIS 2017 fellowship grant (received by Fatema Al-Rashed). Author contribution as follow; FA, ZA, and MA participated in performing experiments, collecting and analyzing data. FA participated in writing manuscript. JT reviewed and critically commented on manuscript, and RA conceived the idea, guided research study, provided material support, procured funds, wrote and edited and approved the manuscript for submission.

\section{Disclosure Statement}

The authors declare no conflict of interests.

\section{References}

- 1 Hotamisligil GS: Inflammation, metaflammation and immunometabolic disorders. Nature 2017;542:177185.

2 Rodrigues KF, Pietrani NT, Bosco AA, Campos FMF, Sandrim VC, Gomes KB: IL-6, TNF- $\alpha$, and IL-10 levels/ polymorphisms and their association with type 2 diabetes mellitus and obesity in Brazilian individuals. Arch Endocrinol Metab 2017;61:438-446.

- 3 Ziegler-Heitbrock L: The CD14+CD16+ blood monocytes: their role in infection and inflammation. J Leukoc Biol 2007;81:584-592.

- 4 Vieira-Potter VJ: Inflammation and macrophage modulation in adipose tissues. Cell Microbiol 2014;16:1484-1492.

5 Ahmad R, Al-Roub A, Kochumon S, Akther N, Thomas R, Kumari M, Koshy MS, Tiss A, Hannun YA, Tuomilehto J, Sindhu S, Rosen ED: The Synergy between Palmitate and TNF- $\alpha$ for CCL2 Production Is Dependent on the TRIF/IRF3 Pathway: Implications for Metabolic Inflammation. J Immunol 2018;200:3599-3611.

6 Kang YE, Kim JM, Joung KH, Lee JH, You BR, Choi MJ, Ryu MJ, Ko YB, Lee MA, Lee J, Ku BJ, Shong M, Lee KH, Kim HJ: The Roles of Adipokines, Proinflammatory Cytokines, and Adipose Tissue Macrophages in Obesity-Associated Insulin Resistance in Modest Obesity and Early Metabolic Dysfunction. PLoS One 2016;11:e0154003. 


\section{Cellular Physiology Cell Physiol Biochem 2019;52:397-407

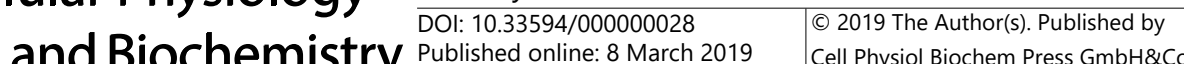 and Biochemistry Published online: 8 March 2019 Cell Physiol Biochem Press GmbH\&Co. KG \\ Al-Rashed et al.: TNF- $\alpha$ Activates Monocytes via ACSL1}

- 7 Hotamisligil GS: Mechanisms of TNF-alpha-induced insulin resistance. Exp Clin Endocrinol Diabetes 1999;107:119-125.

8 Kanter JE, Kramer F, Barnhart S, Averill MM, Vivekanandan-Giri A, Vickery T, Li LO, Becker L, Yuan W, Chait A, Braun KR, Potter-Perigo S, Sanda S, Wight TN, Pennathur S, Serhan CN, Heinecke JW, Coleman RA, Bornfeldt KE: Diabetes promotes an inflammatory macrophage phenotype and atherosclerosis through acyl-CoA synthetase 1. Proc Natl Acad Sci U S A 2012;109:E715-724.

9 Wray GM, Foster SJ, Hinds CJ, Thiemermann C: A cell wall component from pathogenic and non-pathogenic gram-positive bacteria (peptidoglycan) synergises with endotoxin to cause the release of tumour necrosis factor-alpha, nitric oxide production, shock, and multiple organ injury/dysfunction in the rat. Shock 2001;15:135-142.

10 Al-Rashed F, Kochumon S, Usmani S, Sindhu S, Ahmad R: Pam3CSK4 Induces MMP-9 Expression in Human Monocytic THP-1 Cells. Cell Physiol Biochem 2017;41:1993-2003.

11 Sindhu S, Al-Roub A, Koshy M, Thomas R, Ahmad R: Palmitate-Induced MMP-9 Expression in the Human Monocytic Cells is Mediated through the TLR4-MyD88 Dependent Mechanism. Cell Physiol Biochem 2016;39:889-900.

12 Ahmad R, Shihab PK, Jasem S, Behbehani K: FSL-1 induces MMP-9 production through TLR-2 and NF- $\kappa B$ / AP-1 signaling pathways in monocytic THP-1 cells. Cell Physiol Biochem 2014;34:929-942.

- 13 Kochumon S, Wilson A, Chandy B, Shenouda S, Tuomilehto J, Sindhu S, Ahmad R: Palmitate Activates CCL4 Expression in Human Monocytic Cells via TLR4/MyD88 Dependent Activation of NF- $\kappa B / M A P K / P I 3 K$ Signaling Systems. Cell Physiol Biochem 2018;46:953-964.

14 Wouters K, Gaens K, Bijnen M, Verboven K, Jocken J, Wetzels S, Wijnands E, Hansen D, van Greevenbroek M, Duijvestijn A, Biessen EA, Blaak EE, Stehouwer CD, Schalkwijk CG: Circulating classical monocytes are associated with CD11c. Sci Rep 2017;7:42665.

15 Ciapaite J, van Bezu J, van Eikenhorst G, Bakker SJ, Teerlink T, Diamant M, Heine RJ, Krab K, Westerhoff HV, Schalkwijk CG: Palmitate and oleate have distinct effects on the inflammatory phenotype of human endothelial cells. Biochim Biophys Acta 2007;1771:147-154.

16 Xu H, Barnes GT, Yang Q Tan G, Yang D, Chou CJ, Sole J, Nichols A, Ross JS, Tartaglia LA, Chen H: Chronic inflammation in fat plays a crucial role in the development of obesity-related insulin resistance. J Clin Invest 2003;112:1821-1830.

17 Zhu X, Tu Y, Chen H, Jackson AO, Patel V, Yin K: Micro-environment and intracellular metabolism modulation of adipose tissue macrophage polarization in relation to chronic inflammatory diseases. Diabetes Metab Res Rev 2018;34:e2993.

- 18 Yamamoto H, Yoshida N, Shinke T, Otake H, Kuroda M, Sakaguchi K, Hirota Y, Toba T, Takahashi H, Terashita D, Uzu K, Tahara N, Shinkura Y, Kuroda K, Nagasawa Y, Nagano Y, Tsukiyama Y, Yanaka KI, Emoto T, Sasaki N, et al.: Impact of CD14. Atherosclerosis 2018;269:245-251.

- 19 Kumari M, Wang X, Lantier L, Lyubetskaya A, Eguchi J, Kang S, Tenen D, Roh HC, Kong X, Kazak L, Ahmad R, Rosen ED: IRF3 promotes adipose inflammation and insulin resistance and represses browning. J Clin Invest 2016;126:2839-2854.

20 Shoelson SE, Lee J, Yuan M: Inflammation and the IKK beta/I kappa B/NF-kappa B axis in obesity- and dietinduced insulin resistance. Int J Obes Relat Metab Disord 2003;27:S49-52.

21 Weigert C, Brodbeck K, Staiger H, Kausch C, Machicao F, Häring HU, Schleicher ED: Palmitate, but not unsaturated fatty acids, induces the expression of interleukin- 6 in human myotubes through proteasomedependent activation of nuclear factor-kappaB. J Biol Chem 2004;279:23942-23952.

22 Kageyama A, Matsui H, Ohta M, Sambuichi K, Kawano H, Notsu T, Imada K, Yokoyama T, Kurabayashi M: Palmitic acid induces osteoblastic differentiation in vascular smooth muscle cells through ACSL3 and NFкB, novel targets of eicosapentaenoic acid. PLoS One 2013;8:e68197. 